\title{
Femtosecond Surface Vibrational Spectroscopy of CO Adsorbed on Ru(001) during Desorption
}

\author{
Mischa Bonn,* Christian Hess, Stephan Funk, James H. Miners, Bo N. J. Persson, ${ }^{\dagger}$ Martin Wolf, and Gerhard Ertl \\ Fritz-Haber-Institut der Max-Planck-Gesellschaft, Faradayweg 4-6, 14195 Berlin, Germany
}

(Received 9 December 1999)

\begin{abstract}
Using time-resolved sum-frequency generation spectroscopy, the $\mathrm{C}-\mathrm{O}$ stretch vibration of carbon monoxide adsorbed on a single-crystal $\mathrm{Ru}(001)$ surface is investigated during femtosecond near-IR laser excitation leading to desorption. A large transient redshift, a broadening of the resonance, and a strong decrease in intensity are observed. These originate from coupling of the $\mathrm{C}-\mathrm{O}$ stretch to low-frequency modes, especially the frustrated rotation, that are highly excited in the desorption process.
\end{abstract}

PACS numbers: 68.35.Ja, 33.70.Jg, 78.47.+p, 82.20.Rp

The dynamics of the interaction between molecules and metal surfaces is of fundamental importance in surface science, since these determine key physical and chemical properties - essential in, e.g., catalysis - such as energy transfer and molecular reactivity [1]. Surface chemical dynamics are determined by the time scales of various competing relaxation channels [2]: For a complete understanding of surface reactions, it is essential to know how different vibrational modes of the reactant are coupled to each other and to the substrate.

Time-resolved surface vibrational infrared (IR) spectroscopy [3] offers a powerful means of obtaining such insights. Using picosecond (ps) techniques, information about the dynamics of energy exchange between adsorbed carbon monoxide (CO) and metal substrates has been obtained [4], by monitoring transient band shifts of the $\mathrm{C}-\mathrm{O}$ vibration after optical excitation. The technique has further been used to observe vibrational energy relaxation [5] and reversible transient chemical transformations [6] at surfaces. These experiments were limited to temperatures below those at which desorption of the adsorbate occurs. However, when one is interested in surface reactions, the more relevant situation occurs at higher temperatures, where higher-lying vibrational modes get thermally occupied that play an important role in the surface chemistry.

We present here femtosecond time-resolved vibrational sum-frequency generation (fs-SFG) spectra of $\mathrm{CO}$ molecules adsorbed on a $\mathrm{Ru}(001)$ surface, taken while a significant number $(\sim 50 \%)$ of these molecules is desorbing due to fs laser excitation. With this fs-SFG method, snapshots of the $(\mathrm{C}-\mathrm{O})$ stretch vibration can be taken, under conditions where desorption is occurring (at lattice temperatures transiently exceeding the desorption temperature by over $500 \mathrm{~K}$ ), shedding new light on the dynamics of the desorption process and the coupling of vibrational modes at the surface.

The experiments were carried out in an ultrahigh vacuum chamber (base pressure $1 \times 10^{-10} \mathrm{mbar}$ ) equipped with standard surface science tools. Our commercial laser system produces $800 \mathrm{~nm}, 110 \mathrm{fs}$ pulses of $4.5 \mathrm{~mJ} /$ pulse at $400 \mathrm{~Hz}$, chopped down to $20 \mathrm{~Hz}$ for these experi- ments. One-third of the energy is used to excite the surface ("pump" pulse, typical absorbed fluence $55 \mathrm{~J} / \mathrm{m}^{2}$ [7]), and the remaining energy is used to pump an optical parametric generator/amplifier (OPG/OPA) providing tunable ( $\lambda=2-10 \mu \mathrm{m}$, bandwidth $\sim 150 \mathrm{~cm}^{-1}$ ) IR pulses with an energy of typically $10 \mu \mathrm{J}$ and a duration of $150 \mathrm{fs}$. The portion of the $800 \mathrm{~nm}$ pulse which is not converted into IR in the OPG/OPA-process is spectrally narrowed down to $4 \mathrm{~cm}^{-1}$, and is used in the SFG experiments as the visible (VIS) up-conversion pulse. An extensive description of the complete experimental setup as well as the surface cleaning, preparation, and characterization procedures can be found in Ref. [8].

The surface sensitivity of SFG relies on the fact that the second-order nonlinear susceptibility is nonvanishing at interfaces [9]. This enables the generation of an electric field $\boldsymbol{E}_{\mathrm{SFG}}$ out of two incidents fields $\boldsymbol{E}_{\mathrm{VIS}}$ and $\boldsymbol{E}_{\mathrm{IR}}$, where energy $\hbar \omega$ and parallel momentum $\boldsymbol{k}_{\|}$must be conserved $\left(\omega_{\mathrm{SFG}}=\omega_{\mathrm{VIS}}+\omega_{\mathrm{IR}}\right.$ and $\left.\boldsymbol{k}_{\|}^{\mathrm{SFG}}=\boldsymbol{k}_{\|}^{\mathrm{VIS}}+\boldsymbol{k}_{\|}^{\mathrm{IR}}\right)$. For frequencies $\omega_{\text {IR }}$ within the IR bandwidth resonant with the vibrational transition, the signal is resonantly enhanced [10]. Figure 1 depicts the IR spectrum along with the SFG spectrum of $(\sqrt{3} \times \sqrt{3})$-CO $(0.33 \mathrm{ML})$ on $\mathrm{Ru}$ at $340 \mathrm{~K}$. In these experiments the $690 \mathrm{~nm}$ SFG light $(800 \mathrm{~nm}+5 \mu \mathrm{m})$ is dispersed in a monochromator and detected by an intensified charge-coupled device (CCD) array [8]. Thus we achieve a time resolution that is determined by the duration of the fs pump and IR pulses, and a frequency resolution limited by the spectral width of the VIS $(800 \mathrm{~nm})$ up-conversion pulse and the spectrometer, similar to white-light (broadband) probing [11]. The time resolution is demonstrated in the inset of Fig. 1, depicting the free-induction decay (FID), viz. the spectrally integrated SFG intensity as a function of delay between the IR pulse and the $110 \mathrm{fs}$ (800 nm) pump pulse [12]. The time resolution is better than $450 \mathrm{fs}$ - limited by the finite spatial foci and angle between the pump and probe pulses on the surface $\left(40^{\circ}\right.$ and $75^{\circ}$ with respect to the surface normal). The FID decays with a time constant of $\tau=T_{2} / 2=580 \mathrm{fs}$, corresponding to a linewidth of $\Gamma=9.2 \mathrm{~cm}^{-1}$. Some saturation of the vibrational transition could not be 


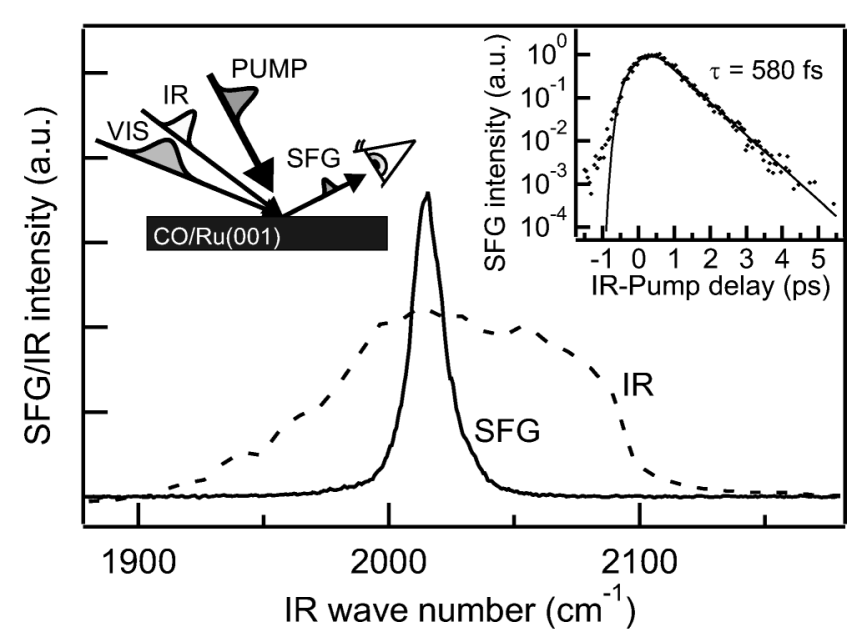

FIG. 1. SFG spectrum of the $\mathrm{C}-\mathrm{O}$ stretching vibration of $(\sqrt{3} \times \sqrt{3})-\mathrm{CO} / \mathrm{Ru}(001)\left(14.3 \mathrm{~cm}^{-1} \mathrm{FWHM}\right)$ at $340 \mathrm{~K}$ and the spectrum of the $150 \mathrm{fs}$ broadband IR pulse (dashed line). The SFG wavelength around $690 \mathrm{~nm}$ is converted to IR frequency. Left inset: Scheme for time-resolved SFG. Right inset: FID of this vibration exhibiting exponential decay with $\tau=580 \mathrm{fs}$, indicating a homogeneous linewidth of $9.2 \mathrm{~cm}^{-1}$. The solid line shows a calculation for a two-level system including spatial and saturation effects. These two data sets demonstrate the $\sim 450$ fs time- and $8 \mathrm{~cm}^{-1}$ spectral resolution (Gaussian instrumental function). The FID was measured with the $110 \mathrm{fs}$ pump pulse $\left(25 \mathrm{~J} \mathrm{~m}^{-2}\right)$ and the broadband IR pulse; the SFG spectrum with the temporally longer VIS up-conversion pulse of $\sim 4 \mathrm{~cm}^{-1}$ spectral width.

avoided, although the sample was moved out of the IR focus to avoid high intensities (IR beam diameter $\sim 0.5 \mathrm{~mm}$ ). Because of dipole-dipole coupling, saturation does not lead to a spectrally separated excited state absorption, but to an increase in linewidth from $\sim 5 \mathrm{~cm}^{-1}$ [13] to $9.2 \mathrm{~cm}^{-1}$, as we determined from IR-power dependent measurements. All the recorded SFG spectra can be very well accounted for by simple Lorentzian line shapes.

Desorption is initiated by excitation of the $\mathrm{CO} / \mathrm{Ru}(001)$ surface with a $(\sim 1.5 \mathrm{~mm}$ diameter $) 800 \mathrm{~nm}$ fs-laser pulse, by which initially only the Ru electrons are heated. Through electron-phonon coupling, the electrons and phonons are equilibrated within $1.5 \mathrm{ps}[2,8]$. It has been demonstrated that $\mathrm{Ru}$ electrons couple inefficiently to $\mathrm{CO}$ and the desorption is triggered by coupling of the $\mathrm{CO}$ to the $\mathrm{Ru}$ phonons $[2,8]$. The right inset of Fig. 2 demonstrates that for an absorbed fluence of $55 \mathrm{~J} \mathrm{~m}^{-2}$, $\sim 50 \%$ of the $\mathrm{CO}$ molecules desorb in a single shot. The figure shows the depletion of the surface coverage due to laser-induced desorption of $\mathrm{CO}$ molecules that, once desorbed, are detected with a fast (time resolution: $<1 \mu \mathrm{s}$ ) mass spectrometer [2,8].

The results of time-resolved SFG experiments for this fluence (temperature before excitation: $340 \mathrm{~K}$ ) are depicted in Fig. 2, where the surface is redosed between the $20 \mathrm{~Hz}$ pump shots over a background of $\mathrm{CO}\left(P_{\mathrm{CO}}=\right.$

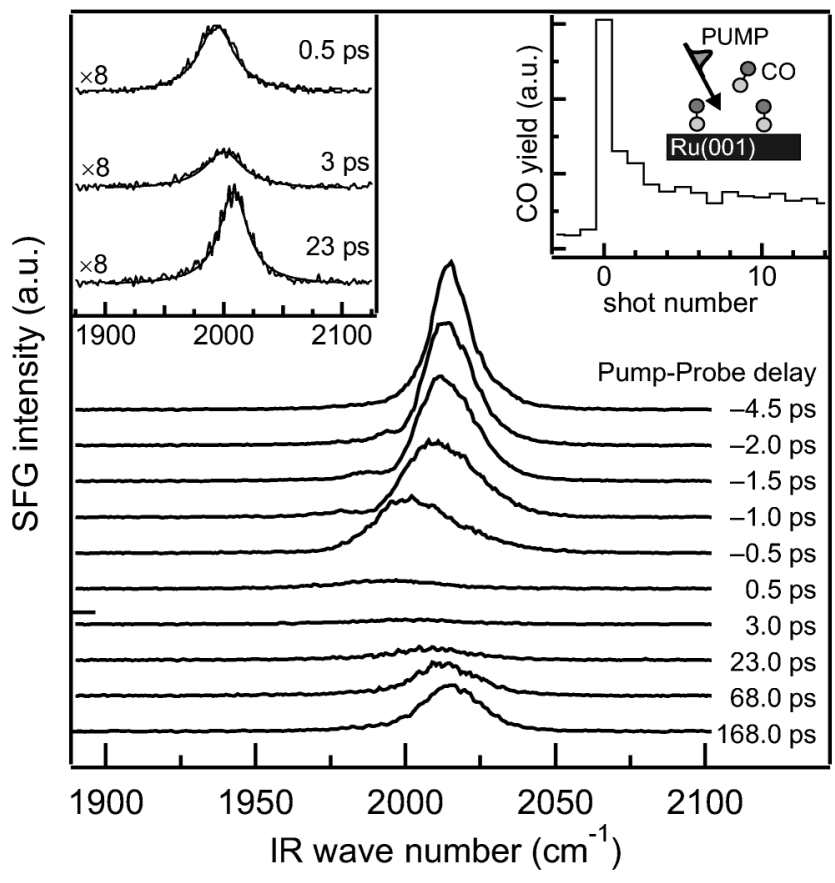

FIG. 2. Transient SFG spectra of the $\mathrm{C}-\mathrm{O}$ stretch vibration while desorption is occurring for delay times indicated in the graph (absorbed pump fluence: $55 \mathrm{~J} / \mathrm{m}^{2}$ ). Lorentzian fits to data in the left inset are shown as dashed lines. Right inset: Depletion curve demonstrating $\mathrm{CO}$ desorption under these excitation conditions. At laser shot number 1 the shutter opens and the surface is irradiated. The $\mathrm{CO}$ yield decreases rapidly after the first shot, indicating a $\sim 50 \%$ desorption probability for the first pulse.

$5 \times 10^{-6}$ mbar), as well as through diffusion of $\mathrm{CO}$ on the surface. The $\mathrm{C}-\mathrm{O}$ stretch vibration displays a very strong transient redshift, as well as a broadening and decrease in integrated intensity. The intensity of the long-time SFG signal $\left(\tau_{\text {delay }}=168 \mathrm{ps}\right)$ is roughly half of the initial intensity, corroborating that a large number of $\mathrm{CO}$ molecules desorb after excitation by the pump pulse, as observed with the direct measurement in the right inset of Fig. 2. The onset of the peak shift at negative delay is not a violation of causality, but rather a consequence of the detection scheme caused by the perturbed free-induction decay [14]: The polarization that is induced by the IR pulse decays with $T_{2}=1.16 \mathrm{ps}$. The effect of the pump pulse can therefore be seen already at negative delay times, when the decaying polarization is perturbed by it. The observed dynamics cannot be explained simply by desorption and subsequent reappearance of $\mathrm{CO}$ molecules through readsorption or diffusion; these mechanisms are negligible for $\mathrm{mm}$ length scales (IR probe diameter) on ps time scales. The data are summarized in Fig. 3, which also depicts the transient band shifts in an experiment with lower fluence $\left(19 \mathrm{~J} \mathrm{~m}^{-2}\right)$ starting with a colder surface $(T=95 \mathrm{~K})$, where no desorption occurs. At this low fluence [7] the peak phonon temperature is $370 \mathrm{~K}$ [15], well below the desorption temperature of $420 \mathrm{~K}$. 


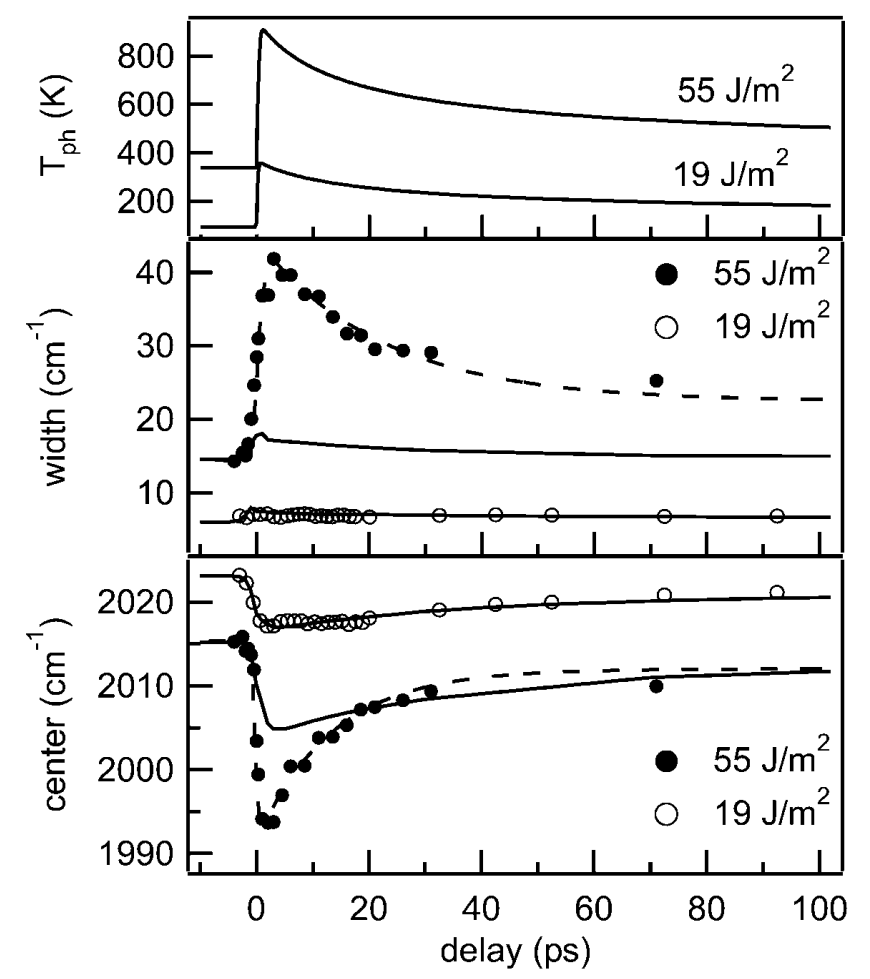

FIG. 3. Temporal evolution of the calculated surface phonon temperature (upper panel) and the measured (circles, dashed lines to guide the eye) and calculated (solid lines) width and center of the $\mathrm{C}-\mathrm{O}$ stretch vibrational resonance. For low $\mathrm{ab}-$ sorbed fluence $\left(19 \mathrm{~J} / \mathrm{m}^{2}\right.$, open circles) the data are very well described by theory. For higher fluences $\left(55 \mathrm{~J} / \mathrm{m}^{2}\right.$, full circles, data of Fig. 2), the theoretical model strongly underestimates the magnitude of the perturbation.

There are three factors that could affect the absorption line of the $\mathrm{C}-\mathrm{O}$ stretch vibration $\nu_{\mathrm{C}-\mathrm{O}}$ in this experiment:

(i) At the high surface temperatures reached in the desorption experiments, one might expect an orderdisorder transition of the ad-layer, resulting in large band shifts and broadening, as has been observed previously for $\mathrm{CO} / \mathrm{Pt}(111)$ [16]. An essential feature of such a transition is the distinct change of the line shape from Lorentzian to Gaussian due to inhomogeneous broadening [16]. However, all the spectra in Fig. 2 can be very well described by Lorentzian line shapes, so that ad-layer disorder is unlikely to be the origin of the observed spectral features.

(ii) The change in surface coverage due to desorption affects the absorption line by a change in dipole-dipole coupling [13]. However, because the surface coverage decreases monotonically in time, the blueshift following the redshift in the observed spectra cannot be accounted for by this mechanism.

(iii) The observations must therefore be explained by transfer of the excitation from the substrate to the adsorbate resulting in "hot" $\mathrm{CO}$ molecules, some of which desorb. The hot $\mathrm{CO}$ molecules will exhibit a different vibrational spectrum, since excitation of low-frequency vibrational modes (such as the $\mathrm{Ru}-\mathrm{CO}$ stretch) may lead to changes in the high-frequency $\nu_{\mathrm{C}-\mathrm{O}}$ mode through anharmonic coupling [17]. Temperature-dependent band shifts and bandwidth changes have indeed been observed for $\mathrm{CO} / \mathrm{Ru}(001)$ [13] due to anharmonic coupling of the $\nu_{\mathrm{C}-\mathrm{O}}$ to the frustrated translational mode at $47 \mathrm{~cm}^{-1}$. At temperatures up to the desorption temperature, the spectra could be very well accounted for by this coupling [13]. We therefore attempted to simulate our SFG data using the same one-mode model.

The expression for the resonant part of the timedependent SFG spectrum $I_{\mathrm{SFG}}\left(\omega ; \tau_{\mathrm{d}}\right)$ at pump-probe delay $\tau_{\mathrm{d}}$ reads

$I_{\mathrm{SFG}}\left(\omega ; \tau_{\mathrm{d}}\right) \propto\left[\int d t \mathrm{e}^{\mathrm{i} \omega t} \int d z P_{\mathrm{IR}}\left(z, t ; \tau_{\mathrm{d}}\right) E_{\mathrm{VIS}}(z, t)\right]^{2}$,

where the spatial integral is over the beam profile, $E_{\mathrm{VIS}}(z, t)$ is the VIS up-conversion field and $P_{\mathrm{IR}}\left(z, t ; \tau_{\mathrm{d}}\right)$ is the IR polarization at the vibrational transition, proportional to the off-diagonal element $\rho_{12}$ of the density matrix, whose time evolution is given by the optical Bloch equations [18]. To obtain the time-dependent width $\Gamma(t)$ and center frequency $\omega_{\mathrm{CO}}(t)$ of the $\mathrm{C}-\mathrm{O}$ vibration, used in the optical Bloch equations, we first calculate the transient substrate phonon temperature $T_{\mathrm{ph}}$ following pulsed laser excitation by means of the two-temperature model [15]. Subsequently, a friction-model calculation allows the calculation of the time-dependent thermal occupation of the $47 \mathrm{~cm}^{-1}$ mode characterized by an adsorbate temperature $T_{\text {ads }}$ [19] by coupling this mode to the phonon heat bath with a coupling time of $\tau_{\mathrm{ph}}=1.0 \mathrm{ps}$ derived from previous experiments [2]. The temperature dependence of $\Gamma\left(T_{\mathrm{ads}}\right)$ and $\omega_{\mathrm{CO}}\left(T_{\mathrm{ads}}\right)$, determined by anharmonic coupling (coupling parameter $\delta \omega=-3 \mathrm{~cm}^{-1}$ ) to the parallel frustrated translation and by the dipole-dipole coupling, are then calculated for all relevant temperatures [17]. Combining these with $T_{\text {ads }}(t)$ yields $\Gamma(t)$ and $\omega_{\mathrm{CO}}(t)$. Thus, the modeled SFG spectra take into account spectral diffusion and include spatial and coherence effects caused by the perturbed free-induction decay. Changes in surface coverage during the experiment are neglected in the calculations. From the resulting calculated spectra we determine the linewidth and center frequency at a given delay time, which are shown in Fig. 3. This full calculation is required to get a correct description of the data; extracting the width and center directly from $\Gamma(t)$ and $\omega_{\mathrm{CO}}(t)$ is insufficient.

The upper panel of Fig. 3 depicts the calculated [15] transient surface phonon temperatures for the two fluences used in the calculations. For the low-fluence data, excellent agreement is observed between experiment and theory (Fig. 3). The observed redshift can be explained by a weakening of the $\mathrm{C}-\mathrm{O}$ bond caused by the increasing amplitude of the (thermally excited) frustrated translation, that displaces the $\mathrm{CO}$ molecule from its on-top position 
towards a bridge position, where it has a lower vibrational frequency. The good agreement between the one-mode theory and our low-fluence experiment has been observed previously in transient band shifts experiments [4] and $T$-dependent IR data [13]. In contrast, for the high-fluence data, apparently a regime is entered where the assumption of coupling to a single mode is no longer adequate: the (maximal) observed change in width is an order of magnitude larger than predicted by theory, indicating that other modes of higher frequency determine the line shape. Indeed, for the high temperatures reached in the experiment, also the frustrated rotational mode $\nu_{\text {rot }}$ at $412 \mathrm{~cm}^{-1}$ and the $\mathrm{Ru}-\mathrm{CO}$ stretch vibration $\nu_{\mathrm{Ru}-\mathrm{CO}}$ at $453 \mathrm{~cm}^{-1}$ [20] will be thermally excited.

The effect of coupling to the metal-CO mode is negligible, since the anharmonic coupling to the $\mathrm{C}-\mathrm{O}$ stretch is only $\delta \omega \approx 2 \mathrm{~cm}^{-1}$ [21]. The theory predicts an increase in the resonance frequency $\nu_{\mathrm{C}-\mathrm{O}}$ of only $2 \mathrm{~cm}^{-1}$ when the temperature is increased from 0 to $900 \mathrm{~K}$, while the contribution to the linewidth is below $1 \mathrm{~cm}^{-1}$ at $900 \mathrm{~K}$. Also, excitation of $\nu_{\mathrm{Ru}-\mathrm{CO}}$ will cause a blueshift of the peak, rather than a redshift, since, upon a weakening of the $\mathrm{Ru}-\mathrm{CO}$ bond, the $\mathrm{C}-\mathrm{O}$ stretch frequency will approach the gas-phase value of $2130 \mathrm{~cm}^{-1}$.

On the other hand, the contribution to the linewidth and frequency shift from the frustrated rotation $\nu_{\text {rot }}$ is much larger than for the metal-CO mode. The linewidth of $\nu_{\text {rot }}$ has been studied (for $T<200 \mathrm{~K}$ ) using IR-spectroscopy, and it is found to depend strongly on temperature [20]. Assuming that this effect is due to two-phonon emission, one expects a linear temperature dependence of the linewidth at high temperatures. Using this result a calculation reproduces the observed maximal peak broadening of the $\mathrm{C}-\mathrm{O}$ mode for $\delta \omega \approx-30 \mathrm{~cm}^{-1}$. Anharmonicities of this magnitude have been observed for other $\mathrm{CO}$ chemisorption systems [17]. We therefore conclude that coupling to $\nu_{\text {rot }}$ plays a crucial role, in addition to coupling to the frustrated translational mode.

In conclusion, we demonstrate for $\mathrm{CO} / \mathrm{Ru}(001)$ that ultrafast energy transfer at an adsorbate-covered metal surface can be studied by recording vibrational spectra of the adsorbate under conditions where reaction is occurring. Our results call for a quantitative theoretical treatment that should further clarify the coupling mechanisms determining the surface chemistry. These experiments open the way for observing chemical reactions in real time through the vibrations of reactants at the surface and for testing the concept of thermal equilibrium underlying the theory of rate processes.
This work was supported in part by the Deutsche Forschungsgemeinschaft through SFB 450. M. B. thanks the European Union (TMR Contract No. ERBFMBICT982971) for financial support.

*Present address: Leiden Institute of Chemistry, P.O. Box 9502, 2300 RA Leiden, The Netherlands.

Electronic address: m.bonn@chem.leidenuniv.nl

†Permanent address: IFF, Forschungszentrum Jülich, D-52425 Jülich, Germany.

[1] R. R. Cavanagh et al., J. Phys. Chem. 97, 786 (1993).

[2] J. A. Prybyla, H. W. K. Tom, and G. D. Aumiller, Phys. Rev. Lett. 68, 503 (1992); M. Bonn et al., Science 285, 1042 (1999).

[3] P. Dumas et al., Surf. Rev. Lett. 6, 255 (1999); Laser Spectroscopy and Photochemistry on Metal Surfaces, edited by H.-L. Dai and W. Ho (World Scientific, Singapore, 1995).

[4] T. A. Germer et al., Phys. Rev. Lett. 71, 3327 (1993); J.P. Culver et al., Chem. Phys. Lett. 214, 431 (1993); A. Bandara et al., Surf. Sci. 427-428, 331 (1999).

[5] M. Morin, N. J. Levinos, and A. L. Harris, J. Chem. Phys. 96, 3950-3956 (1992); P. Guyot-Sionnest et al., Phys. Rev. Lett. 64, 2156 (1990).

[6] A. Bandara et al., J. Phys. Chem. B 102, 5951 (1998).

[7] The reported fluences were corrected to reproduce the $\nu_{\mathrm{CO}}$ shift for $T<400 \mathrm{~K}$ and are consistent with the fluences determined from the beam profile [8].

[8] S. Funk et al., J. Chem. Phys. (to be published).

[9] Y. R. Shen, Nature (London) 337, 519 (1989).

[10] L. J. Richter, T. P. Petralli-Mallow, and J. C. Stephenson, Opt. Lett. 23, 1594 (1998).

[11] J. Glownia et al., Top. Appl. Phys. 70, 1 (1992).

[12] J. C. Owrutsky et al., J. Chem. Phys. 97, 4421 (1992).

[13] P. Jakob and B. N. J. Persson, Phys. Rev. B 56, 10644 (1997).

[14] T. A. Germer et al., J. Chem. Phys. 101, 1704 (1994).

[15] S. I. Anisimov, B. L. Kapeliovich, and T. L. Perel'man, Zh. Eksp. Teor. Fiz. 66, 776 (1974) [Sov. Phys. JETP 39, 375 (1974)].

[16] E. Schweizer et al., Surf. Sci. 213, 49 (1989).

[17] B. N. J. Persson, F. M. Hoffmann, and R. Ryberg, Phys. Rev. B 34, 2266 (1989).

[18] R. W. Boyd, Non-Linear Optics (Academic Press, San Diego, 1992), p. 191.

[19] M. Brandbyge et al., Phys. Rev. B 52, 6042 (1995); L. M. Struck et al., Phys. Rev. Lett. 77, 4576 (1996).

[20] P. Jakob, J. Chem. Phys. 108, 5053 (1998).

[21] P. Jakob and B. N. J. Persson, Phys. Rev. Lett. 78, 3503 (1997). 